\title{
Analysis and Evaluation of the TCP/IP Protocol Stack of LINUX $^{*}$
}

\author{
Guo Chuanxiong, and Zheng Shaoren \\ Institute of Communications Engineering \\ Yudao Street, Nanjing, China \\ Email: xguo@ieee.org
}

\begin{abstract}
With the rapid progress of high-speed network technology, the communication speed between hosts will reach giga $b / s$. So it is important to investigate the traditional TCP/IP protocol stack to find out whether it is suitable for high-speed communication and can provide QoS or not. Based on this consideration, the LINUX TCP/IP stack's structure, the buffer management, the procedures of sending and receiving data, and the interaction with the OS are analyzed in this paper. A probing node method is proposed to investigate the internal structure of the stack. With this method, the throughput of the stack, the time consuming of each layer and the queuing of the IP layer at high speed are tested. Our experiments show that the throughput of TCP/IP stack of LINUX can reach $360 \mathrm{Mbit} / \mathrm{s}$ at the sending side and $150 \mathrm{Mbit} / \mathrm{s}$ when the localhost device is used at bidirection, the protocol stack can be improved to support higher speed; the percentages of the various costs of TCP/IP protocol suite have changed greatly, ZERO-COPY and ZERO-SCAN techniques can not improved the performance dramatically; there is no queuing at the receiving side of the IP layer, so FCFS scheduler is enough, but more sophisticated packet scheduler such as WFQ is needed at the sending layer of the IP layer to provide QoS in the future.

Index terms-LINUX, High-speed Networks, TCP/IP,
\end{abstract}

Probing node, Throughput, Queuing

\section{INTRODUCTION}

The dramatic increase of the network bandwidth and the requests for quality of service(QoS) are two characteristics of the modern network. The speed between hosts has increased to $100 \mathrm{M}, 155 \mathrm{M}$ and even to $1 \mathrm{Gbit} / \mathrm{s}$, at the same time the support of QoS is becoming an important direction in the field of network protocol. Multiservices such as voice, data and real-time video will be supported in

This work was supported by the China High Technology R\&D

Project (the 863 Project) under contract no. 863-300-02-04-99. the future network. So QoS should be provided in the future network with the rapid progress of the network bandwidth.

To analyze the traditional TCP/IP protocol stack to find out the costs of the components and give methods to improve the performance of the protocol is always a hot issue. [4] Analyzed the overhead of the TCP protocol. [7] gave methods to improve the performance of the UDP. [6] studied the TCP/IP stack of SUNOS4.1. [8,9] promoted two ways to implement the TCP/IP protocol in the user space and gave advantages of each method. $[9,15]$ studied the zero-copy and zerochecksumming technology to improve the performance of the protocol. But with the improvement of the capability of the CPU and the transmission speed, and the changes of the network hardware, the behavior of each layer of TCP/IP is changing. For example, with the progress of the hardware and architecture of the computer, the efficiency of the device driver of the network has been improved about 1000 times, and the speed of memory copying has been improved dramatically too. These improvements change the percentages of the cost of each protocol layer. Considering the above reasons, the TCP/IP protocol stack is analyzed and evaluated to find out whether it is suitable for high speed communication and can provide QoS or not. The Linux is chosen as the research object for its widely distribution and open source policy.

Linux is a phenomenon of Internet. It develops and expands itself with the help of Internet. It has a lot of advantages such as powerful developing environment, friendly GUI, widely available application software, good support for a 
wide range of hardware, etc. It is popular because of its open source code and free available applications. The kernel of Linux supports a lot of CPU platforms, and it is a real multitask OS that provides virtual memory protection, demand loading and powerful network function. The network function is a very important part of the Linux kernel. More then $30 \%$ of the Web server on the Internet are constructed on Linux.

The network function of Linux has the following characteristics: it provides BSD SOCKET interface for user applications, it supports network protocols such as IP, IPX, AppleTalk, X.25 etc, it supports a lot of low layer protocols such as Ethernet, token ring, FDDI, Frame Relay and ATM, it supplies device drivers for almost all the Ethernet network controllers, and it has rich IP functions such as IP filtering, IP masquerading, IP alias, and IP accounting.

There are lots of documents introducing and analyzing the Linux kernel such as [4], [10], but the deep analysis of the TCP/IP protocol of Linux is still not available. This paper mainly focuses on the analysis and evaluation of the TCP/IP protocol stack of Linux. In the second part, the structure of the TCP/IP protocol is analyzed with the focus on the general architecture of the protocol stack, the buffer management, the initialization of the protocol stack, the procedure of sending and receiving data, and the interaction with the operation system. The probing method and the design of the probing node are described in the third part. The TCP/IP protocol stack is tested and analyzed in the fourth part, mainly focus on the throughput of the stack, the time cost of each layer, and the queuing of the IP layer. We conclude the paper and describe the future work in the fifth part. The version of Linux kernel we use in the experiments is 2.0.34.

\section{The Structure Of The Linux TCP/IP Protocol STACK}

The protocol stack of Linux is a part of the kernel and is embedded in the kernel code. The structure of the network protocol makes it possible

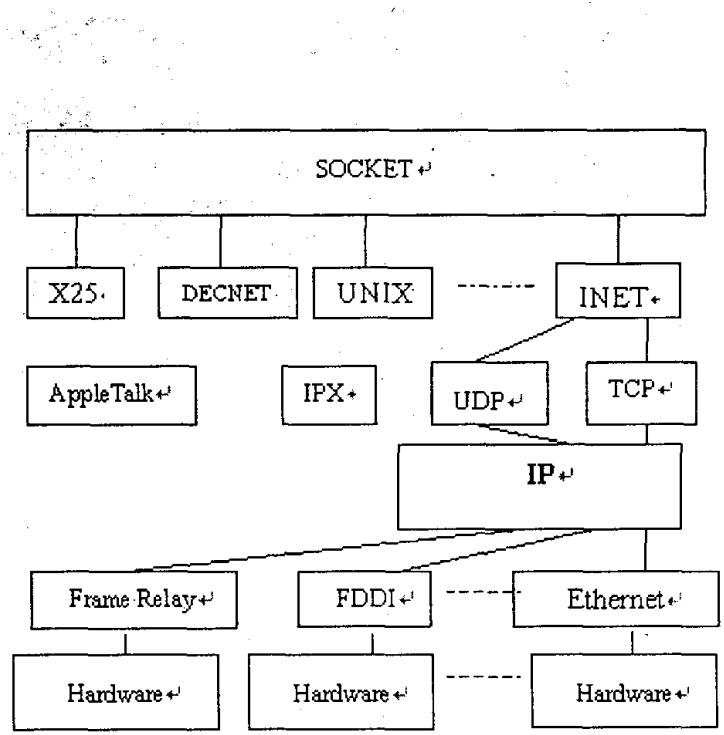

Fig. 1 . architecture of the protocol-stack of Linux * to support lots of protocols at the same time. In this paper, only the TCP/IP protocol stack is analyzed and evaluated. In the following of the paper, if there is no special explaination, the protocol stacks means the TCP/IP stack.

Figure 1 is the architecture of the network structure of Linux. This architecture makes it possible to support multi-network protocol in the Linux kernel. The TCP/IP protocol is made up of 5 layers, the SOCKET layer, the INET layer, the TCP (UDP) layer, the IP layer and network device driver layer. The SOCKET layer is the interface between application programs and lower layer, it covers the difference of various network protocols for application programs. Various lower layer protocols are converged at the IP layer, makes the upper layer of IP irrelevant to the lower layer of IP. In the following sections, the SKBUF buffer management is studied first, next, the data sending and receiving procedure of protocol stack is introduced using UDP as an example, then the queuing of the TCP/IP is described, and the interaction with the operating system is discussed at the end of this part.

\section{A. SKBUF Management}


The buffer management of Linux network protocol is called SKBUF [10], which is different from that of MBUF originated from BSD. The little memory block used by MBUF is not used in the SKBUF mechanism. SKBUF uses continuous memory to store the packet as a whole. In layered protocols such as TCP/IP, the head of the upper layer is added to the data when data is passed from upper layer to lower layer, and the head of the lower layer is stripped off when data is passed from lower layer to upper layer. The method used by Linux is to calculate the maximum amount of memory including the length of the headers of various layers needed by the packet. So when packet is passed between layers the only need is to move the pointers of head or tail of the corresponding SKBUF.

The data structure of SKBUF has the following important parameters: head, pointer of the begin of the SKBUF; end, pointer of the end of the SKBUF; data, pointer of the begin of the valid data of SKBUF; tail, pointer of the end of the valid data in SKBUF. There are also some routines which manipulate the SKBUF: skb_put, puts the data at the end of SKBUF; skb_push, puts the data at the begin of SKBUF; skb_pull, moves the pointer to the valid data backward, it can be used to omit the header of the lower layer at the begin of the packet. Since the SKBUF does not need to manage the links as that of MBUF, it is more efficient than MBUF.

\section{B. The Sending And Receiving Procedures Of $T C P / I P$}

The procedures of sending and receiving data are almost the same for TCP and UDP of the Linux TCP/IP protocol, The only difference is the processing methods of the TCP and UDP layer. So the UDP is chosen to illustrate the procedure for its simplexes. Figure 2 illustrates the sending and receiving procedure. The sending and receiving procedure can be divided into 5 parts as described below:

1) SOCKET layer. It is the interface between user applications and the kernel. It implements the socket system calls provided by the Operation System. The SOCKET layer invokes corresponding lower layer functions with the parameters provided by user programs. For TCP/IP protocol suite, the lower layer of SOCKET is INET.

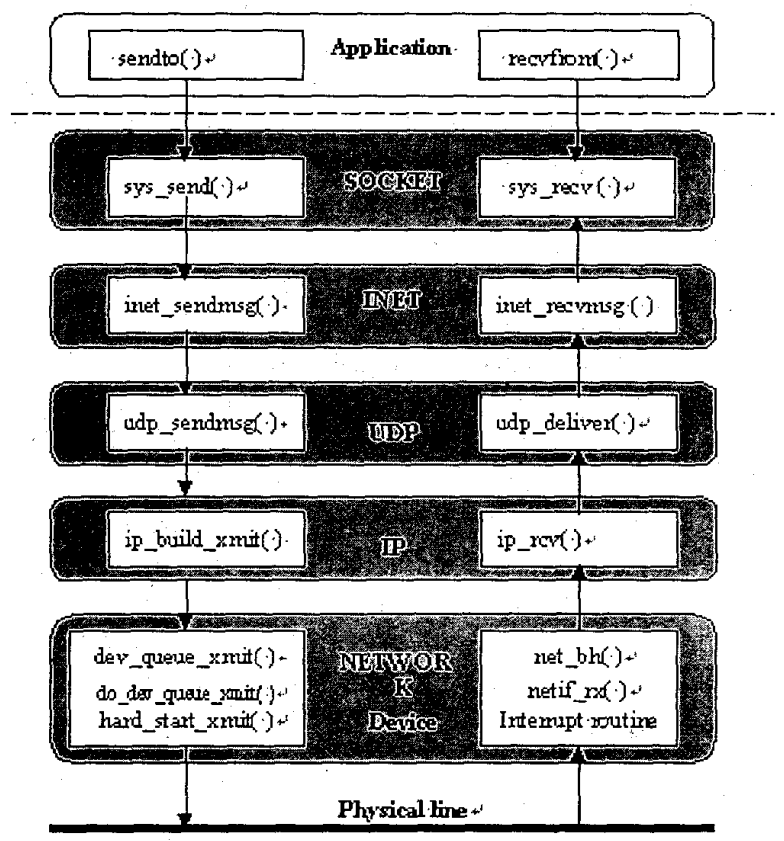

Fiz. 2. Proodures for sending and receiring packets of UDP .

2) INET layer. Its function is to provide a united interface for TCP and UDP.

3) TCP, UDP layer. The TCP and UDP protocols are implemented here. TCP is much more complex than UDP for TCP is a connection oriented transport protocol while UDP is connectionless.

4) IP layer. It implements the IP protocol. The IP layer is the key component of the TCP/IP protocol stack. Its main jobs are to segment and reassemble IP packets, create the SKBUF, lookup the routing table, queue and buffer the IP packets, schedule and forward the IP packet etc. Other IP functions such as IP accounting, IP filtering, IP alias, IP masquerading are all realized in the IP layer.

5) Network device driver layer. Its function is very simple, sends the data from IP layer to the line and receives the data from line and hands it to the IP layer. Linux has abundant device drivers for various network device controllers.

It is notable that the layered principle is not obeyed strictly in Linux. The TCP layer does some of IP's work such as the looking-up of the routing table, the segmentation of the packet, and some of the UDP's work such as the calculation of the 
checksum are done in the IP layer by callback routines provided by UDP.

The other difference between the implementation of TCP and UDP is that in UDP the sending data of the user is copied from user space to kernel space in the IP layer by a callback routine provides by UDP layer while in TCP the copying is done in the TCP layer.

\section{Queuing In The TCP/IP Protocol Of LINUX}

TCP/IP is a kind of store-and-forward protocol, so there will be queues in the protocol stack. The location and the length limitation of the queues, the treatment of the overflowed packet, and the scheduler of the queuing packets will effect the efficiency of the protocol, and also determine whether the protocol can provide QoS or not.

Figure 3 is the queuing model of the TCP/IP protocol of Linux. Queuing exists in two places in the sending and receiving side of the protocol stack respectively. As to TCP protocol, there are two queues at the sending side which are located at TCP layer and network device layer, and two queues at the receiving side which are located at IP layer and TCP layer. The queues of UDP are different from that of TCP, UDP does not have queue at UDP layer because it does not have any means of flow control. The queues at the TCP (UDP) layer are based on the total number of bytes and the queues at the IP and device driver layer are based on the number of packets. The queues at TCP (UDP) are for buffering and flow control (TCP only). The queue at the sending side of the network device driver is for buffering the data temporally when the packet generation speed of IP layer exceeds the sending speed of the physical device. The maximum packet number is tx_queue_len for each device driver. At the receiving side all the received packets are queued in a backlog queue called backlog to wait to be processed by the receiving IP layer. All the device drivers have a unique output queue themselves but share the only input backlog queue. The maximum length of the input backlog queue is 300 . We will see later that the value is big enough, because there is no queue at the receiving side of the IP layer at all.

The queuing system of the protocol stack is a First Come First Serve system (FCFS). The discarding policy used by the stack now is to discard every packet when the maximum length of the limitation is reached without considering of the contents of the packet. So the TCP/IP protocol stack of Linux is not enough to provide QoS for an integrated service network.

\section{Interaction With The Operating System}

The implementation of the TCP/IP protocol suite needs the support of the Operating System and the efficiency of the protocol stack is related heavily to the Operating System. The Operating System provides many functions for the protocol stack such as scheduling of the protocol, memory

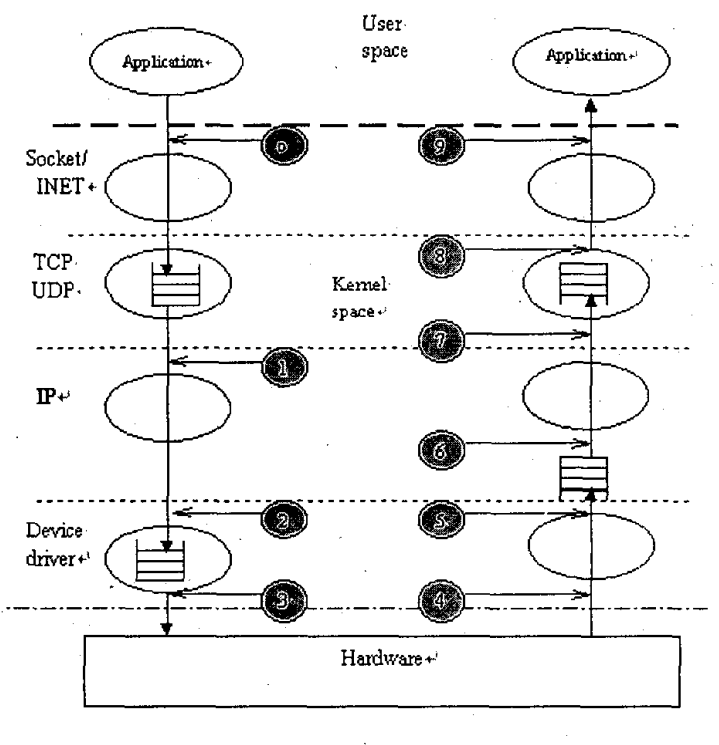

Fig.3. Queuing model of the protocol

management, interrupt management and hardware operations. The scheduling of the protocol has great influence on the efficiency and real-time performance of the protocol. We divide the topic into 3 sections, the initialization of the protocol, the SOCKET system call, and the schedule of the protocol stack.

\section{1) Initialization of the protocol}

The protocol stack is initialized at the end of the initialization of the Linux kernel at the boot time. The main function of the initialization are to initiate the data structure of each layer of the protocol stack, regist the selected protocols such as 
IP, IPX which are selected as parts of the kernel before the compile of the Linux kernel, and establish the relationship between the routines of the lower and the higher layers at the receiving side of the protocol stack. For example, as to UDP/IP suite, the device driver processes the original data received from physical line first, after that, the ip_rcv routine which is registered at the initialization of the protocol will be invoked to continue to process the IP packet, then it will invoke the $u d p \_r c v$ to process the UDP packet. The relationship of the sending routines of each layer is established at SOCKET creation time.

\section{2) The SOCKET system call}

Linux provides a system call named sys_socketcall to provide an interface. to application programs. This interface provides 17 subroutines: sys_socket, sys_bind, sys_listen, sys_accept, sys_connect, sys_send, sys_recv, sys_getsockopt, sys_setsockopt etc. It provides standard BSD Socket interface to realize the separation of the user program and the network functions of the Linux kernel. Generally speaking, the data in the user space should be copied into the kernel space. It is not only needed by the protection mode of the OS, but also by the semantic of SOCKET interface. The kernel and the user programs are different running units, have their own behavior. In order to protect the unity of the data, the user program and the kernel should have a copy of the data of their own independently.

\section{3) The schedule of the protocol}

The efficiency and real-time response of the protocol is related heavily to the schedule of the protocol provided by the operating system. Linux uses a mechanism called bottom_half_handler to deal with works that are related to the device drivers but do not suitable to be processed in the device drivers. The bottom_half_handler related with network protocols is net_bh. The routines in the bottom_half_hander will be scheduled to run by the operating system before the process scheduler and at the end of every system call.

When data is to be sent out, the user program will call the SOCKET interface of the Linux kernel. After being processed by the TCP/IP protocol stack, the "transmission routine in the corresponding network device driver is called to transmit the data. If the device driver is busy in transmitting, the data will be put into the sending queue of the device driver, and will be transmitted by the operating system at the appropriate time.

When packets are received by the hardware, the network controller will invoke an interrupt to the CPU. The operating system will response to the interrupt and fetch the packets into a SKBUF and mark the net_bh as active. The net_bh will be run at appropriate time, and the data will be passed to the TCP/IP protocol stack, after that it will be passed to the user program. In net_bh, Linux will first check that whether there are any packets need to be transmitted, it will transmit the sending packets first if there are any packets in the sending queues, after that it will process the received packets. That is to say, the sending packets are always processed prior to the received packets. Though the schedule of the net_bh relates to the efficiency and real-time performance of the protocol, it is hard to analyze mathematically. This question will be tested in the following experiments.

III The Design Of The Probing Node Based SCHEME

Our goal is to study the performance of the TCP/IP protocol stack as a whole, to study the time costs of each layer and the interaction between the layers of the TCP/IP protocol and the queuing of the IP layer. So the traditional tools which watch, analyze, and measure the external behavior of the protocol such as tcpdump do not meet our purpose. Similar to [6], a probing node based scheme is designed and used to study the internal behavior of the TCP/IP protocol of Linux. A probing node is a piece of code added into the kernel to record the needed information. Some probe nodes are added into the source code of the TCP/IP protocol to memorize information such as the timestamp, the queuing length, and sequence of the packet. Then the information collected by the probing node is analyzed. The probing codes cost CPU time, so they have bad influence on the protocol stack. In order to make the influence as little as possible, the following techniques are used.

1) Only required information is recorded at each probing node. Generally, only the timestamp of the probing node, the sequence of the packet, and the length of the queue (if there is a queue) are recorded. 
2) Only records the packets that meet certain condition. The packets and the socket describers for probing purpose are marked with special flags for the experiments. So only the testing packets will be probed, the other packets will not be affected at all. At the sending side, we add a special flag net_probe_flag in the sock structure relating to the SOCKET describer to trigger the probing code. But at the receiving side, situation is much complex. When the packet is received, it is hard to know that which sock the packet belongs to, so the trigger method of the sending side can not be used at the receiving side. We observe that when packets are passed between the TCP/IP layers, only header is added to or stripped off from the packet, the contents near the tail always unchanged. So when we send test packets, the flag, 0xa5a5a5a5 and the sequence number of the packet are attached to the tail of the packets. When the receiving side receives packets with the flag, it invokes the probing code.

The typical code for the sending side is described as below

\#ifdef NET_PROBE

if(sk->net_probe_flag)

\{

do_gettimeofday(\&temptv);

set_node_value(send_probe2_p, send_probe2_endp, 2, \&temptv, 0,0);

\}

\#endif NET_PROBE

The typical code for the receiving side is described as below, \#ifdef NET_PROBE

do_gettimeofday (\&temptv);

$\mathrm{pp}=($ unsigned int $*)(\mathrm{skb}->$ tail -4$)$;

if $(* p p==0 \times a 5 a 5 a 5 a 5)\{$

$\mathrm{pp}-=1$;

set_node_value(recv_probe1_p,

recv_probe1_endp, 7, \&temptv, *pp,0); \}

\#endif NeT_PROBE

3) The time cost of the probing code is a constant. An area of the memory is reserved when the Linux kernel initializes the memory system. This area is used to store the probing information for each probing node. There is a linear queue for each probing node, and each queue has a pointer points to the first empty place, so the value can be inserted into the queue directly. It has been measured that the time cost of each probing node is 0.4 us. Since the value is a constant, the results of the experiments can be obtained precisely after the subtraction of the time costs of the probing nodes. 4) The collected data is processed off-line.

In order to decrease the infection to the protocol stack of the kernel, the data collection and precession are separated. The data is processed after collecting enough data in the kernel.

Besides the above techniques, all the values are the average of many experiments. The distribution of the probing node is illustrated in Figure 3 . There are 4 probing nodes in the sending side while there are 6 in the receiving side.

\section{Experiments On The TcP/IP Protocol Stack Of} LiNUX

\section{A. The Testing Environment}

The testing environment of this paper is two 128M PII 350 PC connected back to back with twisted wire. The operating system is RedHat 5.1, the kernel version is 2.0.34, and the Ethernet card is Intel Express 100pro with the Intel 82558 network controller. We add 10 probing nodes as described in Figure 3, and reserve 12M RAM in the kernel as the data recording area.

The two computers are running in the multiuser mode with the least background process. This environment applies to all the tests described below if there is no special explains. The throughput of TCP/IP protocol, the time costs of each layer of the protocol stack, and the input and output queues of the IP layer are examined below.

\section{B. The Throughput Of The Protocol}

\section{1) Throughput of $U D P$}

One of the computers acts as the sender, and the other acts as the receiver. The length of the packet is 1024 bytes each. The throughput of UDP at this situation is $93.9 \mathrm{Mbit} / \mathrm{s}$, the physical speed is 97.8Mbit/s after the overhead of the UDP header, the IP header, and the MAC header added to the packet. This speed has reached the limitation of $100 \mathrm{M}$ Ethernet.

\section{2) Throughput of TCP}

The test environment of TCP is the same as . that of UDP. The setsockopt is used to set the 
TCP_NODELAY to the TCP socket at both sides. This makes the packets and ACKs can be sent out in time. Two tests are made, one is the throughputs of the TCP with variable packet length and constant (65536 bytes) sending and receiving buffer, while the other is the throughputs with variable length of the buffers and constant (1024 bytes) length of the packet. The results are shown in Figure 4 and Figure 5.
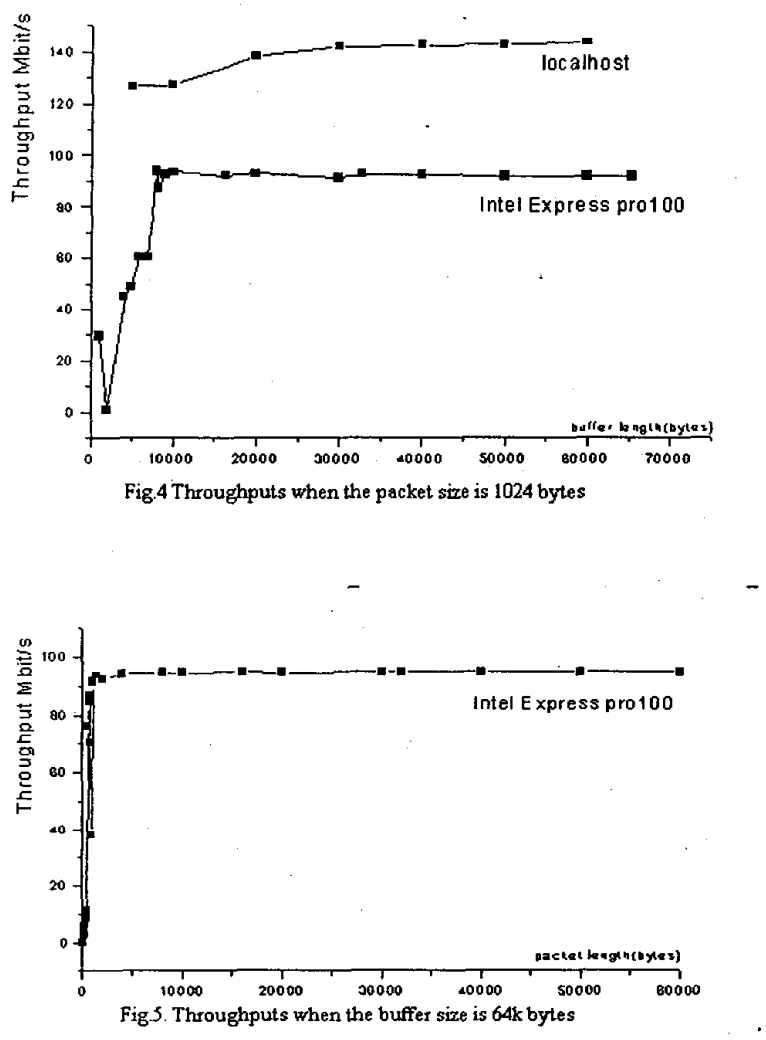

The throughput of TCP reaches $93.9 \mathrm{Mbit} / \mathrm{s}$ with the packet length 1024 bytes and buffer length 7700 bytes, and $94.9 \mathrm{Mbit} / \mathrm{s}$ with the packet length 64000 bytes and buffer length 65536 bytes. These two values correspond to physical value $98.9 \mathrm{Mbit} / \mathrm{s}$ and $96.7 \mathrm{Mbit} / \mathrm{s}$, reach the limitation of $100 \mathrm{M}$ Ethernet too.

It can be concluded that the TCP/IP protocol - stack of Linux can reach the maximum speed that can be provided by the $100 \mathrm{M}$ Ethernet. In the following test, the cost of each part of the protocol stack will be analyzed to find out the maximum processing ability of the stack.

We also find two abnormal phenomena that still need explaination. In Figure 4, when the buffer length is 2048 bytes, the throughput is only $0.82 \mathrm{Mbit} / \mathrm{s}$, greatly lower than the throughput $20.5 \mathrm{Mbit} / \mathrm{s}$ when the buffer length is 1024 bytes. In Figure 5, when the packet length is 900 bytes, the throughput is $37.9 \mathrm{Mbit} / \mathrm{s}$, much lower than the throughputs when the packet length is 800 bytes and 1000 bytes. But these two abnormal phenomena do not appear in the new kernel 2.2.0. it should be the defects of the kernel 2.0.34.

\section{Time Cost Of Each Layer}

This experiment aims to test the cost of each layer of TCP/IP protocol stack of Linux. One HI 350 acts as a sender, sending out a 1024 bytes UDP or TCP packet every 1 second, the other acts as a receiver. The TCP_NODELAY is set for TCP too. The results are the average values of 1000 tests. The results are listed in table I to table V

It is also tested that the memory copy time for 1024 bytes is approximately 1us. From the above table, we know that the SOCKET system call takes about 2 us, and the checksuming time for UDP is 2us. Since DMA is used in the Intel Express 100 pro, there is no need to copy the packet to the network controller by CPU, the sending time of device driver is less than 2us. But it takes about 4us to receive a packet by the device driver. It is because that the procedure for receiving is much complex than that of sending, and there is a memory copy operation in the receiving procedure.

It can be concluded from the above tables that the time cost for sending a 1024 bytes UDP packet when checksum is needed is 18.9 us, for receiving a UDP packet is 35us. So the maximum sending ability of UDP is $433 \mathrm{Mbit} / \mathrm{s}$, the maximum receiving ability for UDP is $234 \mathrm{Mbit} / \mathrm{s}$. The time cost for sending a 1024 bytes TCP packet is $22.5 \mathrm{us,}$ for receiving a TCP packet is 36us. So the maximum sending speed of TCP is $364 \mathrm{Mbit} / \mathrm{s}$, the maximum receiving speed is $228 \mathrm{Mbit} / \mathrm{s}$. It can also be calculated that when the sender and receiver are at the same machine (that is, the localhost device driver is used), the maximum throughput is $150 \mathrm{Mbit} / \mathrm{s}$. This value is consistent with the value 144Mbit/s we got at Figure 4. 
TABLE I

Costs of the sending layers of UDP

(with no checksum to the UDP data) (sum: 18.9 us)

\begin{tabular}{|l|l|l|l|l|}
\hline Item & $\begin{array}{l}\text { System } \\
\text { call }\end{array}$ & $\begin{array}{l}\text { inet_sendmsg- } \\
\text { ip_build_xmit }\end{array}$ & $\begin{array}{l}\text { ip_build_xmit } \\
\text { - speedo_xmit }\end{array}$ & $\begin{array}{l}\text { speedo_xmit } \\
\text { - exit }\end{array}$ \\
\hline Time (us) & 1.8 & 2.3 & 13.2 & 1.6 \\
\hline Percentage & $9.5 \%$ & $12.2 \%$ & $69.8 \%$ & $8.5 \%$ \\
\hline
\end{tabular}

\section{TABLE II}

Costs of the sending layers of UDP

(with checksum to the UDP data) (sum: 17.8us)

\begin{tabular}{|l|l|l|l|l|}
\hline Item & $\begin{array}{l}\text { System } \\
\text { call }\end{array}$ & $\begin{array}{l}\text { inet_sendmsg- } \\
\text { ip_build_xmit }\end{array}$ & $\begin{array}{l}\text { Ip_build_xmit } \\
\text {-speedo_xmit }\end{array}$ & $\begin{array}{l}\text { Speedo_xmit } \\
\text { exit }\end{array}$ \\
\hline Time (us) & 1.8 & 2.8 & 11.4 & 1.8 \\
\hline Percentage & $10.1 \%$ & $15.7 \%$ & $64.1 \%$ & $10.1 \%$ \\
\hline
\end{tabular}

\section{TABLE III}

Costs of the receiving layers of UDP (sum: 35 us)

\begin{tabular}{|l|l|l|l|l|l|l|}
\hline Item & $\begin{array}{l}\text { speedo_rx } \\
- \text { netif_rx }\end{array}$ & $\begin{array}{l}\text { netif_rx } \\
\text { net_bh }\end{array}$ & $\begin{array}{l}\text { net_bh } \\
- \text { ip_rcv }\end{array}$ & $\begin{array}{l}\text { ip_rcv- } \\
\text { udp_rcv }\end{array}$ & $\begin{array}{l}\text { udp_rcv- } \\
\text { inet_recv } \\
\text { msg }\end{array}$ & System call \\
\hline Time (us) & 3.9 & 3.9 & 1.7 & 4.2 & 19.2 & 2.1 \\
\hline Percentage & $11.1 \%$ & $11.1 \%$ & $4.9 \%$ & $12 \%$ & $54.9 \%$ & $6 \%$ \\
\hline
\end{tabular}

\section{TABLE IV}

Costs of the sending layers of TCP (sum: 22.5us)

\begin{tabular}{|l|l|l|l|l|}
\hline Item & $\begin{array}{l}\text { System } \\
\text { call }\end{array}$ & $\begin{array}{c}\text { inet_sendmsg- } \\
\text { ip_queue_xmit }\end{array}$ & $\begin{array}{l}\text { ip_queue_xmit } \\
\text { - speedo_xmit }\end{array}$ & $\begin{array}{c}\text { Speedo_xmit } \\
\text { - exit }\end{array}$ \\
\hline Time (us) & 1.8 & 14.0 & 4.8 & 1.9 \\
\hline Percentage & $8 \%$ & $62.2 \%$ & $21.3 \%$ & $8.5 \%$ \\
\hline
\end{tabular}

\section{TABLE V}

Costs of the receiving layers of TCP (sum: 36 )

\begin{tabular}{|l|l|l|l|l|l|l|}
\hline Item & $\begin{array}{l}\text { speedo_tx } \\
- \text { netif_rx }\end{array}$ & $\begin{array}{l}\text { netif_rx } \\
- \text { net_bh }\end{array}$ & $\begin{array}{l}\text { net_bh- } \\
\text { ip_rcv }\end{array}$ & $\begin{array}{l}\text { ip_rcv- } \\
\text { tcp_rcv }\end{array}$ & $\begin{array}{l}\text { tcp_rcv - } \\
\text { inet_recv } \\
\text { msg }\end{array}$ & $\begin{array}{l}\text { System } \\
\text { call }\end{array}$ \\
\hline Time (us) & 4.0 & 3.8 & 1.5 & 3.8 & 20.8 & 2.1 \\
\hline percentage & $11.1 \%$ & $10.6 \%$ & $4.2 \%$ & $10.6 \%$ & $57.8 \%$ & $5.7 \%$ \\
\hline
\end{tabular}

The time cost for receiving packets and sending packets is different, the receiving side needs more time than that of sending side. That is because the process for receiving is complex than that of sending side, and the receiving process needs more care from the operating system at both the application and the kernel level.

It also can be concluded that the main cost for TCP/IP protocol of Linux is the time for processing the protocol itself. The total time cost needed for kernel memory copying, checksuming, and the system call is $22 \%$ at the sending side of $\mathrm{TCP}, 16.7 \%$ at the receiving side of TCP. The situation is almost the same for UDP. So though techniques such as zero-copy, zero-scan, and the user space protocol stack can improve the performance of the protocol stack, they can not improve the performance dramatically. To optimize the performance of the protocol stack, the processing procedure and the data structure of the protocol stack should be studied.

\section{The IP Queuing At The Maximum Throughput}

This test is designed to study the sending and receiving queues of the IP layer. The queuing at IP layer of the Linux protocol stack is based on FCFS. The study of the behavior of IP queuing, the packet scheduler, and the interaction between the queuing and OS can be a base to provide QoS in the protocol stack in the future.

In this experiment the sender sends UDP packets to the receiver as faster as possible. Figure6, Figure 7 illustrate the queuing at the sending side of 

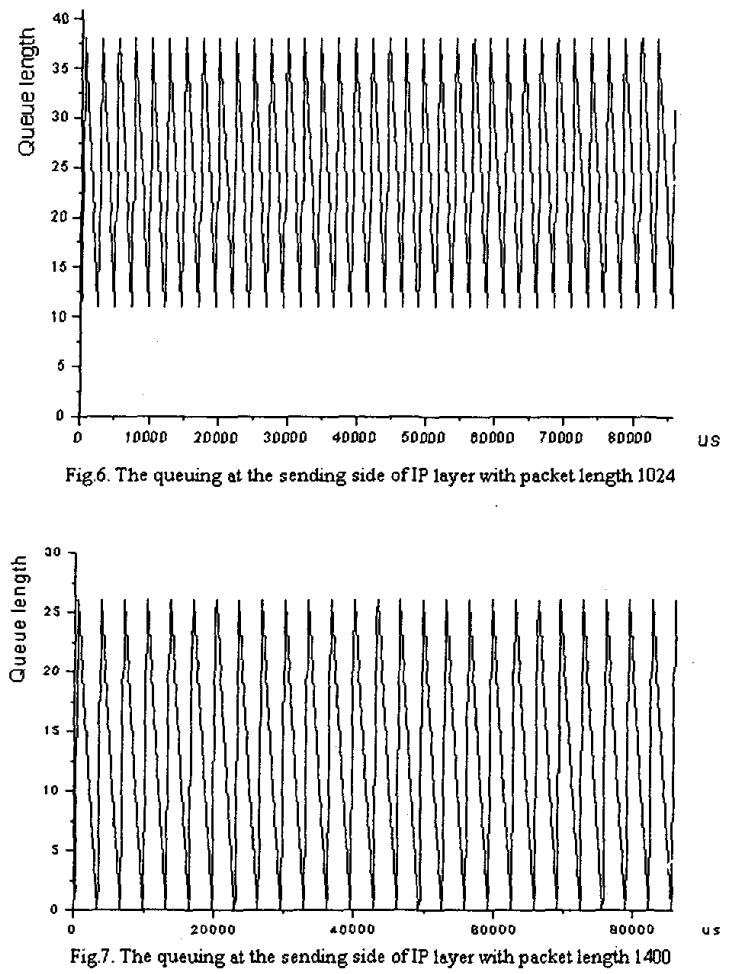

the IP layer when the length of the packet is 1024 and 1400.

From the above figures, we can see an interesting phenomenon, the maximum queuing length does not grow with the growth of the packet length, this indicates that the protocol scheduler of the OS is fairly successful.

Tests also show that there are no queues at the receiving side of the IP layer when the packet length is 1024 and 1400 , and the queuing length varied from 0 to 1 when the packet length is 64 .

The above results were achieved without the competition of other running processes. In orcier to test the queuing of IP layer with the existence of other processes, 1 to 10 processes that are doing mathematical calculations (we call them calculating processes) are introduced into the system where the sender or receiver residents respectively. The results show that at the sender layer, the queuing is the same as that of Fig. 6 and Fig.7 when the sender porcess occupies the CPU, the queuing length is 0 when the calculating processes run. The IP queuing of the receive side is always 0 , but when the number of the calculating processes exceed 6, some of the packets are dropped by the UDP layer because the receiver does not have enough time to process the arrived packets.

We conclude that there is no queuing at the receiving side of the IP layer, but there are queues at the sending side of the IP layer. So there is no need to add any packet scheduler at the receiving side. But some kinds of packet scheduler such as WFQ should be provided to replace the FCFS in the sending side of the IP layer to provide certain QoS in the future.

\section{CONCLUSION AND FUTURE WORK}

The architecture of the TCP/IP protocol stack, the buffer management, the data sending and receiving procedures, the queuing, and the interaction between the protocol stack and OS are studied in this paper. A probing node based method is proposed to analyze the internal structure of the TCP/IP protocol, this method can get the internal parameters of the protocol stack, and has little influence on the protocol. The throughput of the TCP/IP protocol stack, the time cost of each layer, the queuing of IP layer at the throughput of $100 \mathrm{M}$ are examined based on the works above. Briefly, the contributions of the paper are:

1) It gives out the maximum processing ability of TCP and UDP protocol of Linux;

2) Zero-copy and zero-scan technology can only improve the throughput of the TCP/IP protocol at about $20 \%$;

3) There is no queue at the input side of IP layer. But packet scheduler such as WFQ [19]or Time-shift packet scheduler[18] must be added at the output side of IP layer to provide QoS;

4) The Linux Operating System can schedule the protocol stack fairly well.

We will focus on how to improve the processing ability of the protocol stack, and how to provide QoS in the kernel of the Linux in the further study.

\section{REFERENCES}

1. Jon Postel, "User datagram protocol," Reque 
st for comments 768, Aug. 1980.

2. Jon Postel "Transmission control protocol," Request for comments 793, Sept. 1981.

3. Jon Postel, "J Internet protocol," Request for comments 791, Sept. 1981

4. David. A. Rusling, "The Linux kernel," 1998

5. Douglas E. Comer and David L. Stevens, Internetworking with TCP/IP (volume 2) design, implementation, and internals. Beijing, China: Tsinghua University Press, 1998

6. C. Papadopoulos and G.M. Parulkar, "Experimental evaluation of SUNOS IPC and TCP/IP protocol implementation," IEEE/ACM Transactions on Networking, vol.1, no.2, April 1993.

7. Craig Partidge and Stephen Pink, "A faster UDP," IEEE/ACM Transactions on Networking, vol.1, no.4, August 1993.

8. R.Gopalakrishnan and Gurudatte M. Parulkar, "Efficient user-space protocol implementations with QoS guarantees using real-time upcalls," IEEE/ACM Transactions on Networking, vol.6, no.4, April 1998.

9. Peter Steenkite, "Design, implementation, and evaluation of a single-copy protocol stack," Software-Practice and Experience, vol.28, no.7, July 1998.

10. Alan Cox, "Network buffers and memory management," http://www.redhat.com: 8080/HyperNews/get/net/net-intro.html.

11. Art Baker, The Windows NT device driver book: a guide for programmer. Beijing, China: Mechanical Industrial press, April 1998.

12. Xiao Limin and Zhu Mingfa, "Exploiting the capabilities of the interconnection network on dawning-1000," Journal of computer science and technology, no.1, Jan. 1999.

13. Yael Lapid, Raphael Rom, and Moshe Sidi, "Analysis of discarding policies in high-speed networks," IEEE Joumal on selected areas in communications, vol.16, no.5, June 1998.

14. D.D.Clark, V.Jacobson, J. Romkey, and H. Salwen, "An analysis of TCP processing overhead," IEEE commun. mag., vol.27, no.6, p2329, June 1989

15. Gregory G. Finn etc, "The Impact of a ZeroScan Internet checksumming mechanism," SIGCOMM vol.26, no.5, Oct 96.

16. R. Braden, "Requirements for Internet Hosts -Communication Layers," Request for Comments
1122, October 1989.

17. F. Baker, "Requirements for IP Version 4 Routers," Request for Comments 1812, June 1995.

18. Jorge A. Cobb, Mohamed.G. Gonda, and Amal El_nahas, "Time-shift scheduling---fair scheduling of flows in high-speed networks," IEEE/ACM Transaction on Networking, vol.6, no.3, June 1998. 19. Alan Demers, S. Keshav, and S. Shenker, "Analysis and simulation of a fair queueing algorithm," SIGCOMM" 89.

20. W.R.Stevens, UNIX network programming networking APIs: sockets and XTI voll (second edition). Beijing, China: Tsinghua university press, 1998. 